\title{
Optical Vortex Patterns in a Unidirectional ring Oscillator
}

\author{
A. V. Mamaev* and M. Saffman \\ Department of Optics and Fluid Dynamics, Risø National Laboratory, DK-4000 Roskilde, Denmark
}

Received March 14, 1996; accepted April 22, 1996

\begin{abstract}
We describe observation and analysis of optical vortex patterns in a unidirectional ring oscillator with photorefractive nonlinearity. Including field rotation in the resonator leads to novel structures, including counterrotating rings of optical vortices with opposite helicity. A modal analysis is used to explain the observed patterns.
\end{abstract}

\section{Introduction}

Several different mechanisms lead to pattern formation in nonlinear optics. A very general mechanism is the transverse modulational instability of counterpropagating beams [1-3]. The instability occurs in media where the combination of nonlinear phase modulation and linear (diffractive) angle dependent phase retardation leads to instability and oscillation of spatial sidebands. The nonlinear stage of this instability leads to regular transverse patterns, often with hexagonal symmetry $[3,4]$. The instability has been observed in a variety of materials, including atomic vapors [4], liquid crystals [5], photorefractives [6] and organic films [7].

An alternative route to optical pattern formation is to place a nonlinear medium with gain in an optical cavity that supports a spectrum of linear transverse modes. A superposition of the cavity modes is excited and the resulting pattern may be static or dynamic depending on whether or not the modes are frequency degenerate [8-10]. Such a device, a nonlinear gain medium placed in a resonant cavity, is nothing more than a laser. It is therefore no surprise that the resulting patterns are connected closely with the well known Hermite-Gaussian or Laguerre-Gaussian transverse modes of any laser oscillator. Patterns like those described in this report could in principle have been observed in the early days of the laser. Nonetheless, by using optical cavities that are not restricted to oscillation in the lowest, or few lowest, transverse modes, striking and new features emerge.

Patterns observed in lasers supporting many transverse modes are characterized by the appearance of wave front dislocations, or optical vortices. These are points where the intensity goes to zero, while the phase advance on a closed loop about the zero is $2 \pi m$, where $m$ is the charge of the vortex. Optical vortices are well known in linear optics [11] and are also seen as the $\mathrm{TEM}_{01 *}$ or "doughnut" mode of lasers. Solutions of the Maxwell-Bloch equations have demonstrated, in a general context, the existence of dislocations in nonlinear optical media with gain [12]. Dislocations have been observed in a number of experiments

\footnotetext{
* Permament address: Institute for Problems in Mechanics, Russian Academy of Sciences, Vernadsky Prospect 101, Moscow 117526, Russia.
}

with lasers and photorefractive oscillators [13-16]. In particular, the superposition of several frequency shifted modes has led to the observation of moving regular vortex patterns $[17,18]$.

There are some basic differences between pattern formation due to transverse modulational instability and patterns observed in resonant cavities. In the modulational instability case a nonlinear phase shift is necessary for oscillation, whereas in the cavity case it is sufficient to have a medium with nonlinear gain. A nonlinear phase shift may, nevertheless, occur in the cavity case due to frequency shifts between the pump and oscillating fields. These two systems are also distinct as regards the types of patterns they produce. Patterns due to modulational instability may be classified as "mixed" modes whereas patterns observed in resonant cavities are close to "pure" modes. This terminology may be understood as follows. The paraxial wave equation admits Hermite-Gaussian and Laguerre-Gaussian functions as solutions. It is well known that any Hermite-Gaussian [19] or Laguerre-Gaussian function of a given order is selfsimilar upon Fourier transformation. More precisely $\mathscr{F}\left(\left\{H_{n}(x) \mathrm{e}^{-x^{2}}\right\} \sim \mathrm{i}^{n} H_{n}(\tilde{x}) \mathrm{e}^{-\tilde{x}^{2}}\right.$, where $\mathscr{F}$ is the Fourier transform operator, $H_{n}$ is the $\mathrm{n}^{\text {th }}$ Hermite polynomial, and tildes denote conjugate variables. Similarly, the Hankel transform of the Laguerre polynomial mode $L_{p}^{m}\left(r^{2}\right) \mathrm{e}^{-r^{2}} \sim$ $\mathrm{i}^{m} L_{p}^{m}\left(\tilde{r}^{2}\right) \mathrm{e}^{-\tilde{r}^{2}}$. This implies that the intensity pattern due to a single Hermite-Gaussian or Laguerre-Gaussian mode is self-similar in the near and far fields, or at any plane in between. This also applies to superpositions of the form $\sum_{n} H_{4 n} \mathrm{e}^{-x^{2}}$. Likewise, patterns due to mode superpositions with widely spaced values of the mode index will be approximately self-similar in intensity. We refer to such patterns as "pure" modes since they are form invariant under linear propagation. Transverse modulational instability results in "mixed" modes that are not form invariant under propagation.

Oscillators pumped by nonlinear beam coupling in a photorefractive crystal are an attractive system for studying these patterns. The large nonlinearity and narrow gain line of photorefractive two-beam coupling leads to strong frequency pulling such that transverse modes with relative frequency shifts of tens of $\mathrm{Hz}$ are observed, even though the empty cavity modes have natural frequency differences in the $\mathrm{MHz}$ range. The resulting slowly moving patterns can be recorded directly with video cameras. A number of recent works have studied pattern dynamics in photorefractive ring oscillators [14-18]. We describe in section 2 new forms of these patterns obtained in a ring oscillator with field rotation in the cavity. The study of patterns generated in cavities with field rotation was pioneered by Vorontsov, and a huge variety of patterns have been observed [20]. The difference 
here is that we are dealing with a coherent oscillator that is sensitive to the phase of the optical field whereas the field rotation systems studied by Vorontsov were based on incoherent cavities. The possibility of self-consistent modes in such a cavity can be understood from the fact that the generated modes rotate as they propagate. The geometric rotation in the cavity is thus compensated for by diffractive rotation. A modal analysis is given in section 3 where it is shown that the observed patterns are discrete superpositions of Laguerre-Gaussian modes. Section 4 contains some concluding remarks.

\section{Experiment}

The experimental geometry is shown in Fig. 1. The ring resonator was formed from three planar mirrors, a lens of focal length $f=150 \mathrm{~mm}$, a dove prism that can be rotated about its axis, and a photorefractive gain medium. The effective cavity length, accounting for the index of refraction of the prism and the gain medium, was adjusted to $L=600 \mathrm{~mm}$ within an accuracy of 1-2 mm. The cavity was thus approximately concentric. Gain was provided by a $5.2 \mathrm{~mm}$ long crystal of $\mathrm{KNbO}_{3}$ doped with $0.5 \%$ by weight $\mathrm{Fe}$ pumped by a $22 \mathrm{~mW}$ beam from a $532 \mathrm{~nm}$ frequency doubled Nd:YAG. The pump beam was focused to a Gaussian radius of $w_{\mathrm{p}}=0.43 \mathrm{~mm}$ in the crystal. Scattered light seeds unidirectional oscillation in the ring. The orientation of the $\mathrm{KNbO}_{3}$ crystal is chosen such that the oscillating field counterpropagates to the pump beam, and couples to it via formation of a reflection grating oriented along the crystal c-axis. The cavity mirrors $M_{1-3}$ had reflectivities of 100,95 and $50 \%$. The outputs transmitted through the partially refiecting mirrors were projected on a screen using relay lenses to give simultaneous near-and far-field pictures. The near field picture is a magnified image of the field at the right hand surface of the gain medium in Figure 1. The farfield image is its Fourier transform, obtained by propagating for $3 \mathrm{~m}$ in free space. Fine adjustment to the length of the cavity was made by mounting mirror $M_{1}$ on a piezoelectric translator.

The structure of the generated field depends on the cavity Fresnel number which quantifies the number of available degrees of freedom. A concentric resonator is self-imaging (up to a minus sign) and the Fresnel number of the empty resonator is is not well defined. Recalling that the effective

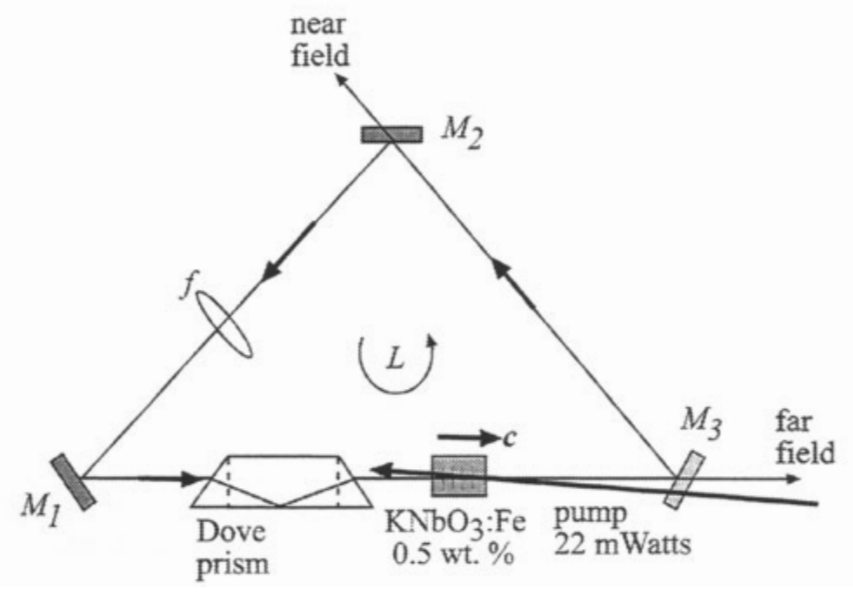

Fig. 1. Experimental geometry. size of the gain medium is fixed by the pump beam we have for the Fresnel number of the resonator with gain medium $\mathscr{F}=4 w_{\mathrm{p}} w_{\text {lens }} /(\lambda L)$, where $w_{\text {lens }}$ is the radius of the clear aperture of lens $f$. This expression applies when the gain medium is located symmetrically opposite the lens, so that the beam waist is located inside the gain medium. For our resonator parameters we find $\mathscr{F} \sim 50$. At such high values of the Fresnel number we observe a generated beam in the form of a spatiotemporally chaotic speckle field [14].

The Fresnel number can be reduced by moving the gain medium away from the plane where the beam waist lies. Referring to Fig. 1 if the counterclockwise distance from lens to crystal is $L_{1}$ and the distance from crystal to lens is $L_{2},\left(L_{1}+L_{2}=L\right)$ we have approximately $\mathscr{F}=$ $\left(4 w_{\mathrm{p}} w_{\text {lens }} /(\lambda L)\right)\left(1-2 L_{1} / L\right)$. For the experimental results reported below $L_{1}$ was adjusted to give $\mathscr{F} \sim 5-15$. Under these conditions we observed regular transverse patterns. These patterns were observed both with $L<4 f$ (stable, near concentric resonator) and $L>4 f$ (unstable, near concentric resonator). Geometric stability is not important here, because the Gaussian profile of the pump beam assures the existence of stable modes for all cavity lengths.

Figures 2 to 4 show examples of the observed patterns recorded with a video camera. Each row shows the far-field (left) and near-field (right) images. The patterns were observed for the same resonator parameters, but slightly different resonator lengths, obtained by adjusting piezoelectric mirror $M_{1}$ or tilting the gain crystal slightly. Patterns a-c in Fig. 2 are modes with a bright center and a ring of 3, 4 and 5 field dislocations respectively. Patterns $d$ and e are more
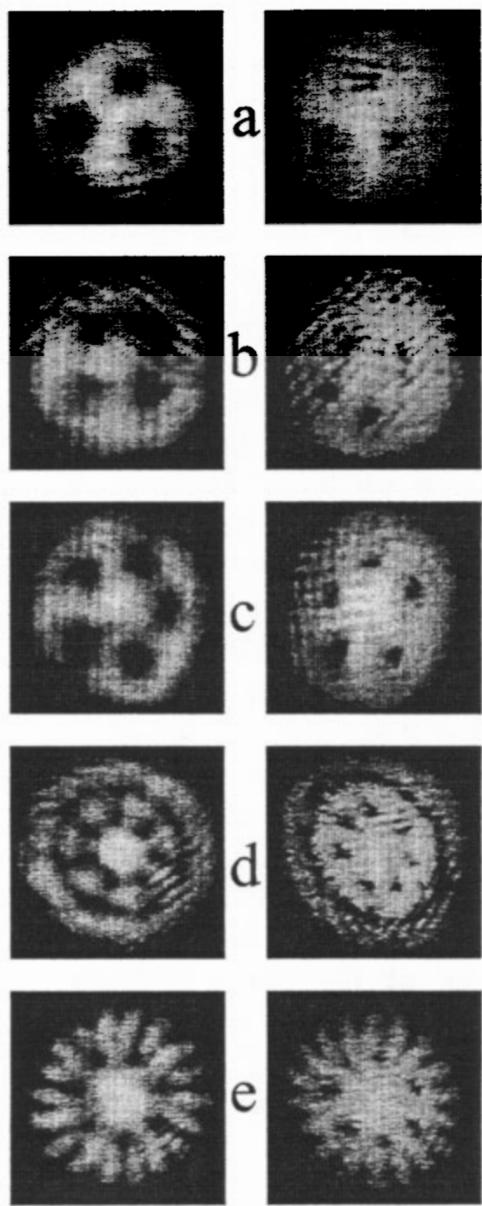

Fig. 2. Patterns with a bright center. 

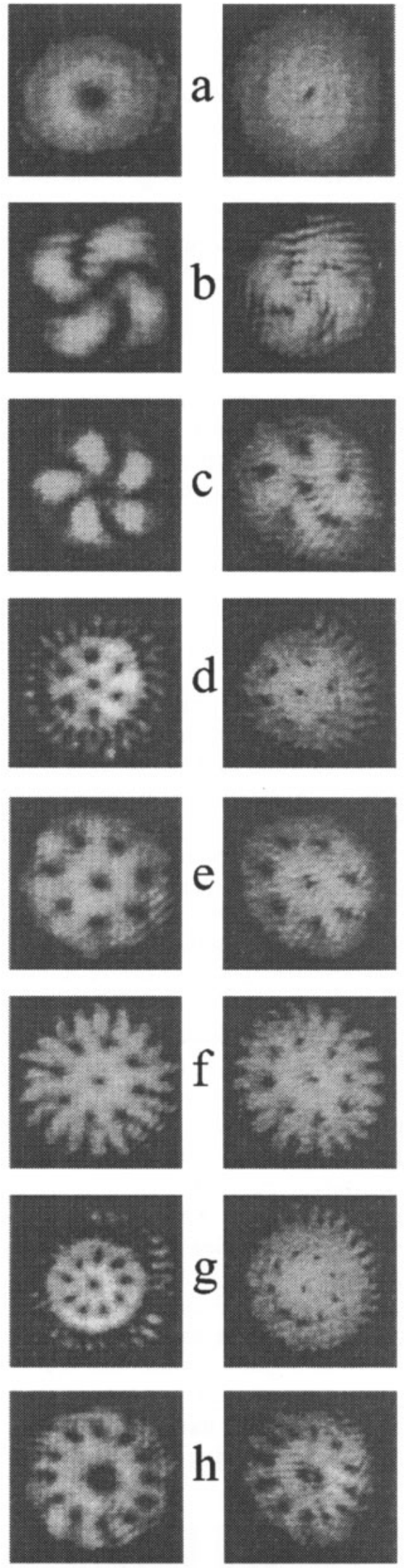

Fig. 3. Patterns with a zero at the center.

complex. Pattern $\mathrm{d}$ has an inner ring of 7 zeroes, a second ring of zeroes, and an outer bright ring. Pattern e has an inner ring of 7 zeroes and an outer ring of 17 zeroes. Figure 3 shows patterns with a zero at the center and a ring of 0,4 , $5,6,7,8,9$ and 12 zeroes in frames a-h respectively. Frame $f$ is very similar to frame e in Fig. 2 (except for different numbers of zeroes), and frames $d$ and $g$ have outer rings
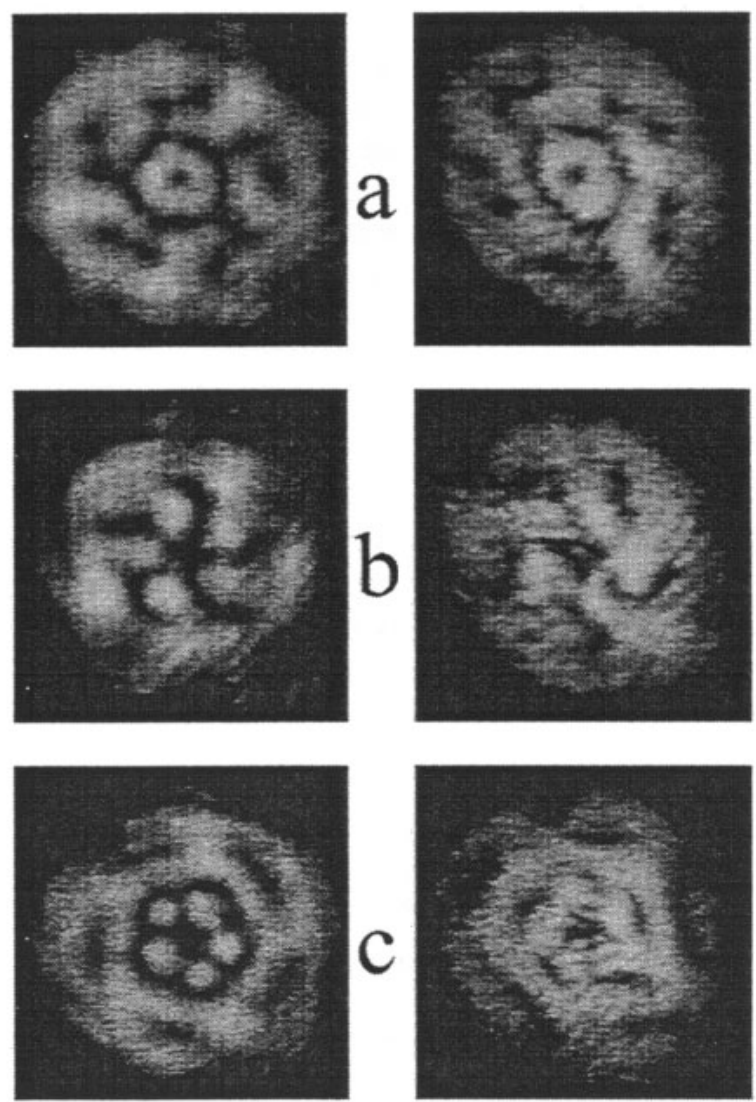

Fig. 4. Patterns with strong pentagonal symmetry.

with close to 30 zeroes. Finally, Fig. 4 shows unusual patterns with strong pentagonal symmetry. The interpretation of the dark spots in these patterns as field dislocations was verified by interferometric observations. The helicity of the vortices on a given ring was the same. The relatively complex patterns with multiple concentric rings were observed only at relatively high values of the cavity Fresnel number $(\mathscr{F} \sim 15)$.

All of these patterns were observed with the dove prism set to give a rotation angle of about $30^{\circ}$. The exact setting of the dove prism was not important. Corresponding patterns were observed for a range of rotation angles. In all cases the patterns were observed to rotate at frequencies of up to several revolutions per second. The speed of rotation depended on fine tuning of the cavity length. This is due to the fact that the rotation of the patterns is due to small relative shifts between the pulled mode frequencies. The direction of rotation was not fixed, and spontaneous switching between rotation directions was seen. Apart from a momentary condition during switching of the rotation direction, the patterns were never observed to stand still. The inner and outer rings in the multiple ring patterns were observed to rotate simultaneously in opposite directions. The patterns could be selected by tuning the length of the resonator. This was in accord with the observations in [16] that the observed mode is determined by the resonance condition of the empty (linear) resonator. More complex spatiotemporal dynamics involving repetitive, periodic switching between a set of transverse modes was also observed.

It is apparent from Figs. 2-4 that the observed patterns exhibit a large degree of self-similarity. As explained in the introduction, self-similarity implies that the patterns may be 
represented accurately by superpositions of a few LaguerreGaussian modes. Only in Fig. 4 do we see patterns that are far from being self-similar. This may be due to the fact that these patterns, in particular, were recorded under conditions where the resonator mode exhibited periodic switching. A related recent experiment where patterns were observed in a linear photorefractive resonator driven by counterpropagating pump beams gave qualitatively different results [21]. In that case the observed patterns were not self-similar.

\section{Modal Analysis}

The linear modes of the resonator can be found using the well known formalism of ray-matrices [22]. When the resonator possesses axial symmetry it is most convenient to use Laguerre-Gauss modes as a set of basis functions for representing the resonator field $A=\sum_{p m} c_{p m} A_{p m}$. These functions form a complete set and may be written in the following normalized form

$$
\begin{aligned}
A_{p m}(r, \phi, z)= & \sqrt{\frac{2^{(1+|m|)} p !}{\pi w^{2}(z)(p+|m|) !}}\left(\frac{r}{w(z)}\right)^{|m|} L_{p}^{|m|}\left(\frac{2 r^{2}}{w^{2}(z)}\right) \\
& \times \mathrm{e}^{-r^{2} / w^{2}(z)} \mathrm{e}^{\mathrm{i} k r^{2} / 2 \rho^{2}(z)} \mathrm{e}^{\mathrm{i}(k z+\psi p m(z))} \mathrm{e}^{\mathrm{i} m \phi .}
\end{aligned}
$$

Here $r$ is the radial coordinate, $\phi$ is the azimuthal coordinate, $L_{p}^{|m|}$ is a generalized Laguerre polynomial, $w(z), \rho(z)$ are the Gaussian width and radius of curvature respectively, and $\psi_{p m}(z)$ is the diffractive phase shift due to propagation. The normalization has been chosen such that

$$
\left\langle A_{p m}, A_{p^{\prime} m^{\prime}}\right\rangle=\int_{0}^{2 \pi} \mathrm{d} \phi \int_{0}^{\infty} \mathrm{d} r r A_{p m} A_{p^{\prime} m^{\prime}}^{*}=\delta_{p p^{\prime}} \delta_{m m^{\prime \prime}}
$$

It is convenient to define the reference plane where the beam waist occurs $(\rho=\infty)$ as $z=0$, in which case the phase shift is given by [22] $\psi_{p m}(z)=(2 p+|m|+1) \tan ^{-1}$ $\left(B /\left(A z_{\mathrm{R}}\right)\right)$, where $A, B$ are the ray-matrix elements for propagation from the reference plane, and the Rayleigh length is $z_{R}=\pi w^{2}(0) / \lambda$. The dependence of the phase shift on the mode index has an important consequence; patterns that are superpositions of modes with different values of $2 p+|m|$ rotate as they propagate. To illustrate this consider the field with $m_{1}$ wave front dislocations given by $A=$ $c_{00} A_{00}+c_{0 m_{1}} A_{0 m_{1}}$. On the circle with radius

$r_{1}=\frac{w}{\sqrt{2}}\left(\frac{c_{00}}{c_{0 m_{1}}} \sqrt{\left|m_{1}\right| !}\right)^{\left(1 /\left|m_{1}\right|\right)}$

there are $m_{1}$ dislocations at angles

$\phi_{n}^{(1)}=\frac{2 n+1}{m_{1}} \pi$,

where $n=0, \ldots m_{1}-1$. Free space propagation through a distance $l$ gives $\psi_{p m_{1}}=\left(2 p+\left|m_{1}\right|+1\right) \tan ^{-1}\left(l / z_{\mathrm{R}}\right)$. The field dislocations thus rotate clockwise looking along the direction of propagation by an angle $\delta \phi=\operatorname{sign}\left(m_{1}\right) \tan ^{-1}\left(l / z_{\mathrm{R}}\right)$. This rotation was verified experimentally for $m_{1}=2$ in [23].

Some of the observed patterns are characterized by concentric rings of dislocations. To describe this situation we write the field in the form $A=A_{00}+A_{0 m_{1}}+A_{0 m_{2}}$, with $m_{2}>m_{1}$. The resulting mode has an inner circle containing $\left|m_{1}\right|$ zeroes and an outer circle of $\left|m_{2}-m_{1}\right|$ zeroes. For $m_{1}$ and $m_{2}$ sufficiently different the radius of the inner circle is given approximately by eq. (3) while the outer circle has radius

$r_{2} \approx \frac{w}{\sqrt{2}}\left(\frac{c_{0 m_{2}}}{c_{0 m_{1}}} \sqrt{\frac{\left|m_{1}\right| !}{\left|m_{2}\right| !}}\right)^{\left(1 /\left(\left|m_{2}\right|-\left|m_{1}\right|\right)\right)}$

By way of example $m_{1}=8$ and $m_{2}=20$ gives, for equal modal coefficients $c_{0 m}, r_{1} / w \approx 1.37$ and $r_{2} / w \approx 2.65$. The angular positions of the dislocations on the outer ring are given by

$\phi_{n}^{(2)}=\frac{2 n+1}{m_{2}-m_{1}} \pi$,

where $n=0, \ldots m_{2}-m_{1}-1$. The helicity of the dislocations is given by sign $\left(m_{1}\right)$ on the inner ring and sign $\left(m_{2}\right)$ on the outer ring. Propagation from $z=0$ to $z=l$ results in rotation of the inner and outer circles by

$\delta \phi^{(1)}=\operatorname{sign}\left(m_{1}\right) \tan ^{-1}\left(l / z_{\mathrm{R}}\right)$

and

$\delta \phi^{(2)}=\frac{\left|m_{2}\right|-\left|m_{1}\right|}{m_{2}-m_{1}} \tan ^{-1}\left(l / z_{\mathrm{R}}\right)$

respectively. When $m_{1}$ and $m_{2}$ have the same sign both rings rotate by the same amount, and when $m_{1}$ and $m_{2}$ have opposite signs the rotation in the outer ring is smaller.

Consider now the question of self-consistent modes in the resonator with field rotation. Denoting the rotation angle introduced by the dove prism by $\phi_{p}$ we require $\phi_{p}$ $+\delta \phi^{(1)}=l_{1}\left(2 \pi / m_{1}\right)$ on the inner circle and $\phi_{p}+\delta \phi^{(2)}=$ $l_{2}\left(2 \pi /\left(m_{2}-m_{1}\right)\right)$ on the outer circle, where $l_{1}, l_{2}$ are the number of pattern periods rotated in each round trip. Using eqs. $(7,8)$ it is possible to find integers $m_{1}$ and $m_{2}$ which satisfy these two conditions.

It should be noted that besides the rotation due to diffractive propagation there may also be a contribution due to the formation of a twisted holographic grating inside the nonlinear medium. Particularly if the cavity length is adjusted to be perfectly concentric there will be no diffractive contribution to the pattern rotation.

Figure 5 gives an example of a representation of a pattern with a central dislocation, plus two rings of vortices, using the modal superposition $A=A_{0,1}+A_{0,9}+A_{0,-20}$. The calculated pattern is similar in a qualitative sense to the observed structure, although details of the relative sizes of the dark and bright regions are not accurately reproduced. There are several reasons for expecting less than perfect agreement. For the first we have assumed a modal superposition with equal weighting coefficients. In reality the
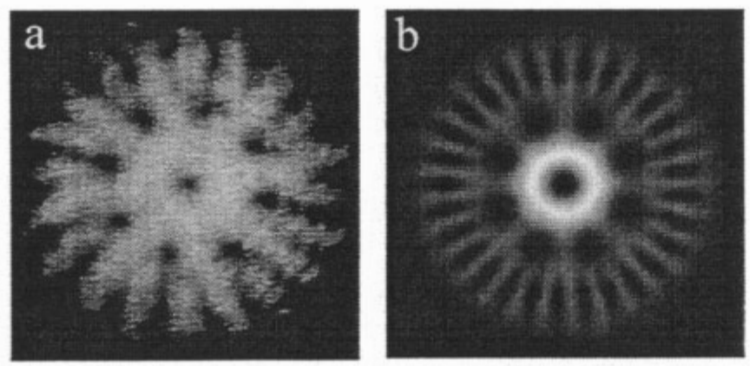

Fig. 5. Comparison of experimental observation (a) and modal superposition (b). 
modes participate with unequal weights, since their frequencies are slightly different (this is what gives rise to the temporal rotation), and the modes thus receive slightly different amplification. The modal functions in eq. (1) all have the same characteristic spatial scale $w$. This follows from linear resonator theory. In reality nonuniform saturation in the wings of the Gaussian pump beam is expected to give the different modes different effective diameters $w \rightarrow w_{p m}$.

\section{Conclusions}

In summary we have observed patterns characterized by concentric rings of optical vortices in a ring resonator with field rotation. The patterns are closely related to linear resonator modes. In particular there is a strong degree of selfsimilarity between the near-and far-field intensity distributions. The observed patterns were non-stationary. Both steady circling motion of the vortices, including counterrotation of concentric rings, and periodic switching between patterns, were observed.

\section{Acknowledgements}

This work was supported by a grant from the Danish Natural Science Research Council.

\section{References}

1. Vlasov, S. N. and Talanov, V. I., in: "Optical Phase Conjugation in Nonlinear Media”, (Edited by V. I. Bespalov) (Institute of Applied Physics, USSR Academy of Sciences, Gorki, 1979).
2. Firth, W. J. and Paré, C., Opt. Lett. 13, 1096 (1988).

3. Grynberg, G., Opt. Commun. 66, 321 (1988).

4. Grynberg, G. et al., Opt. Commun. 67, 363 (1988).

5. Macdonald, R. and Eichler, H. J., Opt. Commun. 89, 289 (1992).

6. Honda, T., Opt. Lett. 18, 598 (1993).

7. Glïckstad, J. and Saffman, M., Opt. Lett. 20, 551 (1995).

8. Brambilla, M. et al., Phys. Rev. A, 43, 5090 (1991).

9. Brambilla, M. et al., Phys. Rev. A, 49, 1427 (1994).

10. Coates, A. B. et al., Phys. Rev. A, 49, 1452 (1994).

11. Baranova, N. B., Zel'dovich, B. Ya., Mamaev, A. V., Pilipetsky, N. F. and Shkunov, V. V., Pis'ma Zh. Eksp. Teor. Fiz. 33, 206 (1981) [JETP Lett., 33, 195 (1981)].

12. Coullet, P., Gil, L. and Rocca, F., Opt. Commun. 73, 403 (1989).

13. Weiss, C. O., Physics Reports 219, 311 (1992).

14. Arecchi, F. T., Giacomelli, G., Ramazza, P. L. and Residori, S., Phys. Rev. Lett. 65, 2531 (1990).

15. Liu, S. R. and Indebetouw, G., J. Opt. Soc. Am B, 8, 1507 (1992).

16. Hennequin, D., Dambly, L., Dangoisse, D. and Glorieux, P., J. Opt. Soc. Am B, 11, 676 (1994).

17. Vaupel, M. and Weiss, C. O., Phys. Rev. A, 51, 4078 (1995).

18. Staliunas, K., Tarroja, M. F. H., Slekys, G., Weiss, C.O. and Dambly, L., Phys. Rev. A, 51, 4140 (1995).

19. Titchmarsh, E. C., "Introduction to the Theory of Fourier Integrals", 2nd ed., (Oxford University Press, Oxford, 1959).

20. Akhmanov, S. A., Vorontsov, M. A. and Ivanov, V. Yu., Pis'ma Zh. Eksp. Teor. Fiz. 47, 611 (1988) [JETP Lett. 47, 707 (1988)].

21. Mamaev, A. V. and Saffman, M., Opt. Commun., vol. 128, 281 (1996).

22. Anan'ev, Y. A., "Laser Resonators and the Beam Divergence Problem", (Adam Hilger, Bristol, 1992).

23. Basistiy, I. V., Bazhenov, V. Yu., Soskin, M. S. and Vasnetsov, M. V., Opt. Commun. 103, 422 (1993). 